\title{
Clinical Outcomes in Iraqi Patients with Rheumatoid Arthritis Following Earlier or Later Treatment with Etanercept
}

\author{
Nizar Al-Ani (D) \\ Faiq Gorial' \\ Dina Yasiry ${ }^{2}$ \\ Fadya Al Derwibee ${ }^{2}$ \\ Yasameen Abbas Humadi (iD ${ }^{3}$ \\ Nancy Sunna ${ }^{4}$ \\ Ali AlJabban ${ }^{5}$ \\ 'Rheumatology Unit, College of \\ Medicine, University of Baghdad, \\ Baghdad, Iraq; ${ }^{2}$ Department of \\ Rheumatology, Baghdad Teaching \\ Hospital, Baghdad, Iraq; ${ }^{3}$ College of \\ Medicine, Al-Nahrain University, \\ Baghdad, Iraq; ${ }^{4}$ Medical Department, \\ Pfizer, Amman, Jordan; ${ }^{5}$ Medical \\ Department, Pfizer, Baghdad, Iraq
}

Correspondence: Ali Allabban

Pfizer, Building 5, Street 5I, Sec. 6II, AIDawoodi, Baghdad, Iraq

Tel +9647706056226

Email AliAbdulmohsinAbdulkareem.

AlJabban@pfizer.com
Purpose: The development of evidence-based guidelines on early pharmacotherapeutic treatment of rheumatoid arthritis (RA) could be useful in Middle Eastern nations striving to improve outcomes in patients with this chronic, debilitating disease. Evidence obtained from local populations should inform such guidelines and therefore our aim was to use realworld data to evaluate the clinical responses of Iraqi patients with RA who received earlier or later treatment with the TNF inhibitor etanercept.

Patients and Methods: Data from patients registered in the Iraq National Center of Rheumatology database from May 2012 to December 2018, inclusive, were analyzed retrospectively. Inclusion criteria were age $\geq 18$ years, meeting the ACR/EULAR 2010 criteria for RA, referral for etanercept treatment, and $\geq 1$ year of follow-up after etanercept initiation. Patients were excluded if they had received another biologic for RA. Included patients were categorized according to two separate stratifications: whether duration of RA symptoms prior to etanercept initiation was $\leq 10$ or $>10$ years ( 10 years represented the mean duration for the entire analysis population); and according to whether duration of RA symptoms prior to etanercept initiation was $\leq 1,>1$ to $\leq 4,>4$ to $\leq 10,>10$ to $\leq 20$, or $>20$ years. The evaluated outcomes were mean change from baseline in Clinical Disease Activity Index (CDAI) and 28-joint Disease Activity Score (DAS28) after 1 year of etanercept treatment.

Results: A total of 979 patients were included. CDAI and DAS28 were significantly reduced $(p<0.001$ for both) after 1 year of etanercept treatment irrespective of whether duration of RA symptoms prior to treatment was $\leq 10$ or $>10$ years. Patients with RA symptoms for $\leq 1$ year prior to etanercept initiation showed a significant reduction in CDAI after 1 year of treatment $(\mathrm{p}=0.01)$.

Conclusion: Iraqi patients with RA who received earlier treatment with etanercept had superior outcomes compared with those who received later treatment.

Keywords: autoimmunity, biologics, delayed treatment of rheumatoid arthritis, early treatment of rheumatoid arthritis, real-world data, TNF inhibitors

\section{Introduction}

Initiating pharmacotherapy soon after the onset of rheumatoid arthritis (RA) reduces the progression of joint damage and improves long-term outcomes. ${ }^{1-3}$ The European League Against Rheumatism (EULAR) therefore recommends that treatment with a disease-modifying anti-rheumatic drug (DMARD) should be initiated as soon as a diagnosis of RA is made because untreated disease does not remit spontaneously. ${ }^{4}$ The clinical outcomes of patients with RA have improved considerably since the tumor necrosis factor inhibitor (TNFi) class of biologic 
DMARDs was introduced, with etanercept being the first in $1998 .^{5}$ Etanercept has a clinical profile that has been extensively characterized in both randomized controlled trials and real-world studies conducted over a period of more than 20 years.

Evidence from the Middle East suggests that the management of patients with RA in this region could be further optimized. ${ }^{6-8}$ In Iraq there are national guidelines on the treatment of RA that are endorsed by the Ministry of Health, but which are not formally published, and Iraqi rheumatologists are guided by these in addition to publications from EULAR and the American College of Rheumatology (ACR). Patients with RA in Iraq receive methotrexate as a first-line treatment and become eligible to initiate a biologic, such as etanercept, if they have established, severe, and active symptoms of RA after $\geq 3$ months of treatment with methotrexate $(25 \mathrm{mg} /$ week; or alternative conventional synthetic DMARD in case of methotrexate intolerance) and $\geq 2$ of the following prognostic factors: smoking, very high erythrocyte sedimentation rate (ESR) and C-reactive protein results, very high rheumatoid factor or anti-citrullinated protein antibody results, radiologic evidence of joint damage, or the presence of nodules or other extra-articular features. The development of new national guidelines on early therapeutic intervention could be one way to improve outcomes in Iraqi patients and this requires evidence obtained from studies in local populations. The aim of the present study was to use real-world data to evaluate the clinical responses of Iraqi patients with RA who received earlier or later treatment with the TNFi etanercept.

\section{Patients and Methods}

\section{Study Design}

This was a retrospective, observational study of real-world patient data entered into the Iraq National Center of Rheumatology database between May 2012 and December 2018, inclusive. Patients were included in the analysis if they were aged $\geq 18$ years, met the ACR/ EULAR 2010 criteria for RA, had been referred for treatment with etanercept, and had $\geq 1$ year of follow up after initiating etanercept. Patients were excluded if they had ever received any biologic agent for RA other than etanercept. Included patients were categorized according to two separate stratifications in order to investigate the relationship between outcomes and the duration of RA symptoms prior to etanercept initiation. The first stratification categorized patients into two groups according to whether the duration of RA symptoms prior to etanercept initiation was $\leq 10$ or $>10$ years, with 10 years representing the mean duration of RA symptoms for the entire analysis population. The second stratification categorized patients into five groups according to the duration of RA symptoms prior to etanercept initiation: $\leq 1,>1$ to $\leq 4,>4$ to $\leq 10,>10$ to $\leq 20$, and $>20$ years.

All of the patients whose data were included in this study provided informed consent. The study and its protocol were approved by the ethics committee of Baghdad Teaching Hospital and all aspects of the study's conduct followed the principles laid out in the Declaration of Helsinki.

\section{Outcomes and Statistical Analysis}

Clinical response to etanercept treatment was assessed by evaluating mean changes in Clinical Disease Activity Index (CDAI) and 28-joint Disease Activity Score (DAS28, calculated using ESR data) from baseline to 1 year after initiation of treatment. Two-sided, independent-sample t-tests were used to assess the statistical significance of clinical responses after 1 year of treatment and Mann-Whitney $U$-tests were used for non-parametric comparisons $\left(\right.$ IBM $^{\circledR}$ SPSS Statistics v22). Results were considered statistically significant if $\mathrm{p}<0.05$ without multiplicity adjustment.

\section{Results}

A total of 979 Iraqi patients with RA were included in the analysis, $813(83 \%)$ of whom were women (Table 1). None of the included patients had ever received a biologic agent for any indication except for receiving etanercept for RA. The mean duration of RA symptoms prior to the initiation of etanercept treatment was 10 years. Patients who initiated etanercept treatment $<10$ years after the onset of RA symptoms were significantly younger and had a significantly shorter mean duration of RA symptoms, as well as significantly lower CDAI and DAS28. A significantly greater proportion of the patients who initiated etanercept treatment $>10$ years after the onset of RA symptoms were women.

In terms of clinical outcomes, CDAI and DAS28 were significantly reduced $(\mathrm{p}<0.001$ for both) after 1 year of etanercept treatment irrespective of whether the duration of RA symptoms prior to treatment was $\leq 10$ or $>10$ years (Figure 1A and B). Patients with RA symptoms for $\leq 1$ year prior to etanercept initiation showed a statistically significant reduction in CDAI after 1 year of treatment $(p=0.01)$ while 
Table I Baseline Characteristics of Iraqi Patients with RA Who Initiated Etanercept Treatment

\begin{tabular}{|c|c|c|c|c|}
\hline Patient Characteristics & $\begin{array}{c}\text { All } \\
\text { Patients, } \\
\mathrm{N}=979\end{array}$ & $\begin{array}{c}\text { Earlier Tx } \\
(\leq 10 \text { Years Since Onset of RA), } \\
n=579\end{array}$ & $\begin{array}{c}\text { Later Tx } \\
\text { (>10 Years Since Onset of RA), } \\
n=400\end{array}$ & $\begin{array}{c}\text { p value } \\
\text { (Earlier vs Later } \\
\text { Tx })\end{array}$ \\
\hline Female, n (\%) & $813(83)$ & $469(8 I)$ & $344(86)$ & 0.046 \\
\hline Age, years & $48.1 \pm 12.1$ & $45.2 \pm 12.2$ & $52.2 \pm 10.6$ & $<0.001$ \\
\hline $\begin{array}{l}\text { Duration of RA symptoms } \\
\text { prior to etanercept Tx, years }\end{array}$ & $10.1 \pm 8.3$ & $4.7 \pm 2.4$ & $18.0 \pm 7.4$ & $<0.001$ \\
\hline CDAI & $27.7 \pm 11.6$ & $27.0 \pm 11.8$ & $28.6 \pm 11.4$ & 0.044 \\
\hline DAS28 & $5.7 \pm 1.2$ & $5.6 \pm 1.2$ & $5.8 \pm 1.2$ & 0.031 \\
\hline Methotrexate Tx, n (\%) & $535(55)$ & $326(56)$ & $209(52)$ & Not significant \\
\hline Prednisolone Tx, n (\%) & $427(44)$ & $247(43)$ & $180(45)$ & Not significant \\
\hline
\end{tabular}

Note: Data are mean \pm standard deviation unless otherwise noted.

Abbreviations: CDAI, Clinical Disease Activity Index; DAS28, 28-joint Disease Activity Score; RA, rheumatoid arthritis; Tx, treatment.

those with a duration of RA symptoms prior to etanercept initiation of $>1$ to $\leq 4,>4$ to $\leq 10,>10$ to $\leq 20$, or $>20$ years showed numerical reductions that did not achieve statistical significance (Figure 2A). The numerical reductions in DAS28 observed in patients with RA symptoms of $\leq 1,>1$ to $\leq 4,>4$ to $\leq 10,>10$ to $\leq 20$, or $>20$ years did not achieve statistical significance after 1 year of treatment (Figure 2B).

\section{Discussion}

The results of our analysis show that Iraqi patients with RA who received treatment with etanercept for 1 year had significant improvements in CDAI and DAS28 irrespective of whether the duration of their RA symptoms prior to treatment was below or above the cohort mean of 10 years. Patients with a mean duration of RA symptoms prior to etanercept initiation of $\leq 1$ year showed a significant improvement in CDAI after 1 year of treatment. Overall, our data provide further evidence of the importance of initiating pharmacotherapeutic treatment of RA soon after symptoms manifest. ${ }^{1-3}$

The mean duration of RA symptoms prior to etanercept initiation was 10 years in the Iraqi cohort evaluated in this analysis, which indicates a considerable delay in the initiation of biologic treatment and this is likely to have influenced the extent of the patients' responses to etanercept. The delay in referral for treatment with etanercept may be due to a lack of awareness of rheumatic disease among the Iraqi public, a lack of knowledge among Iraqi health care professionals, or a combination of both, ${ }^{6-8}$ although it should also be noted that there is very limited access to treatment with biologics for patients with RA in Iraq. Access to this type of treatment is provided solely by the national Ministry of Health and patients with RA who initiate biologics tend to be those who have very severe symptoms and have failed on maximum-dose methotrexate and another DMARD. As well as the delay in referral for etanercept treatment, another noteworthy aspect of the cohort included in our study was that there was a significantly greater proportion of women among the group with a duration of RA symptoms $>10$ years prior to etanercept initiation compared with those with a duration of symptoms $<10$ years, although the difference was modest ( 86 vs $81 \%$; $p=0.46$ ). The reason for this gender difference is unclear and requires further research in order for its basis to be understood and appropriately mitigated.

To our knowledge there have not been any previous publications that describe a cohort of patients with RA of a similar size from Iraq or the wider Middle East region. A Phase 4, open-label study of tocilizumab, a biologic that targets the IL-6 receptor, was conducted across 5 Middle Eastern countries (Bahrain, Iran, Kuwait, Qatar, and the UAE) from 2010 to 2011 and reported a reduction in mean DAS28 from a baseline value of 6.1 to 2.1 after 24 weeks of treatment. ${ }^{9}$ The 95 patients included in the study of tocilizumab had a similar baseline DAS28 to the patients in our study (6.1 vs 5.7), as well as a similar mean age at baseline (44.9 years vs 48.1 years), but the tocilizumab study did not report the mean duration of RA symptoms prior to treatment with tocilizumab and this confounds any comparison of the extent of the treatment effect observed. 


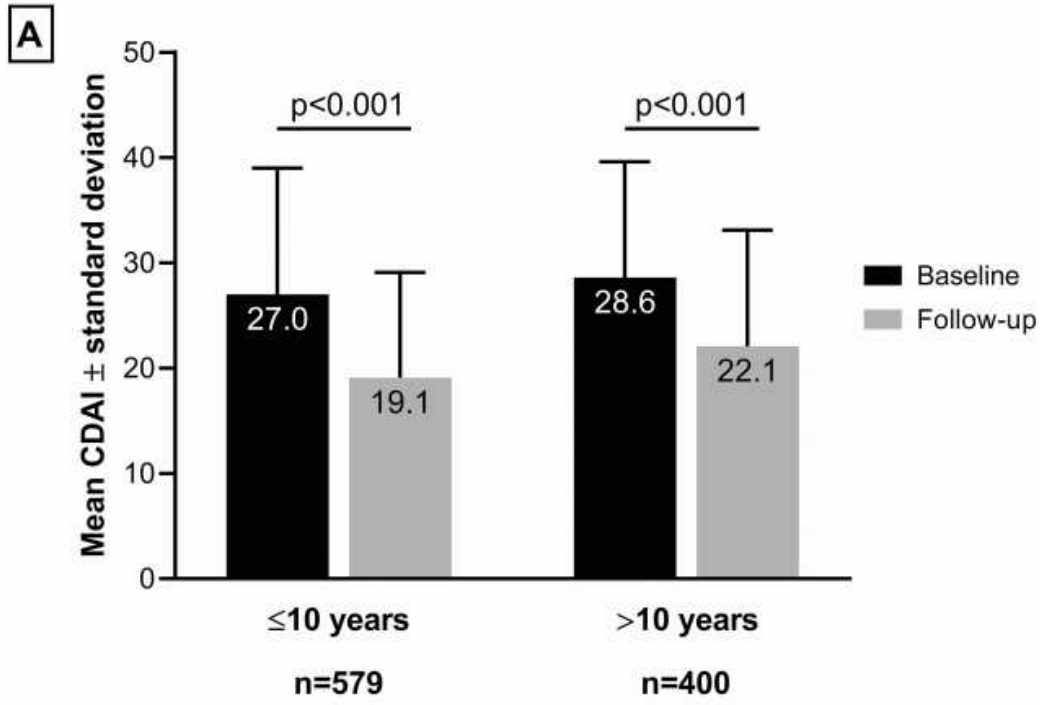

\section{Duration of RA prior to initiation of etanercept treatment}

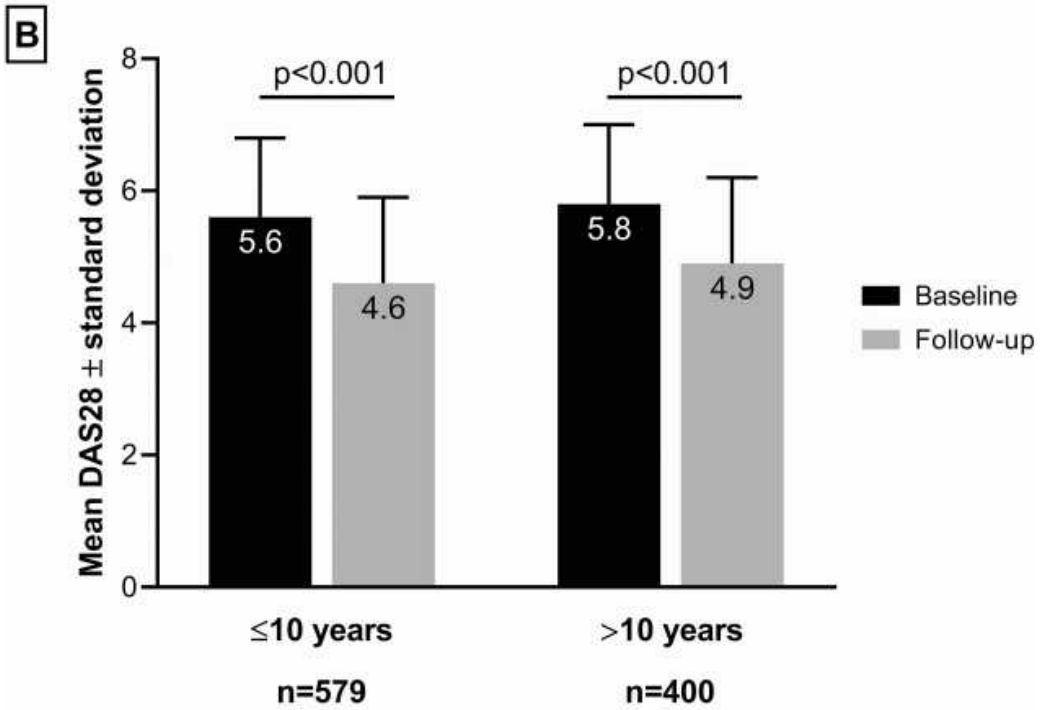

Duration of RA prior to initiation of etanercept treatment

Figure I Change from baseline in (A) CDAI and (B) DAS28 in Iraqi patients stratified according to whether duration of RA symptoms prior to etanercept initiation was less than or greater than the population mean of 10 years.

Abbreviations: CDAI, Clinical Disease Activity Index; DAS28, 28-joint Disease Activity Score.

Our retrospective, observational study has some limitations. First, the data describing the duration of RA symptoms prior to etanercept initiation obtained from the Iraq National Center of Rheumatology database relied on the ability of patients to accurately recollect their onset, which in some cases was a long time. Second, our study focused on outcomes relating to the clinical effectiveness of etanercept and did not evaluate safety or quality-of-life outcomes that are important aspects of any treatment's overall clinical profile. Third, we evaluated CDAI and DAS28 as clinical outcomes but did not investigate changes in the diagnostic imaging of joint degradation, which is a key disease process that treatments for RA aim to arrest as much as possible.

\section{Conclusion}

Iraqi patients with RA who receive earlier treatment with etanercept have superior outcomes compared with those who receive later treatment. Increasing the awareness of RA and the importance of early treatment with a biological 


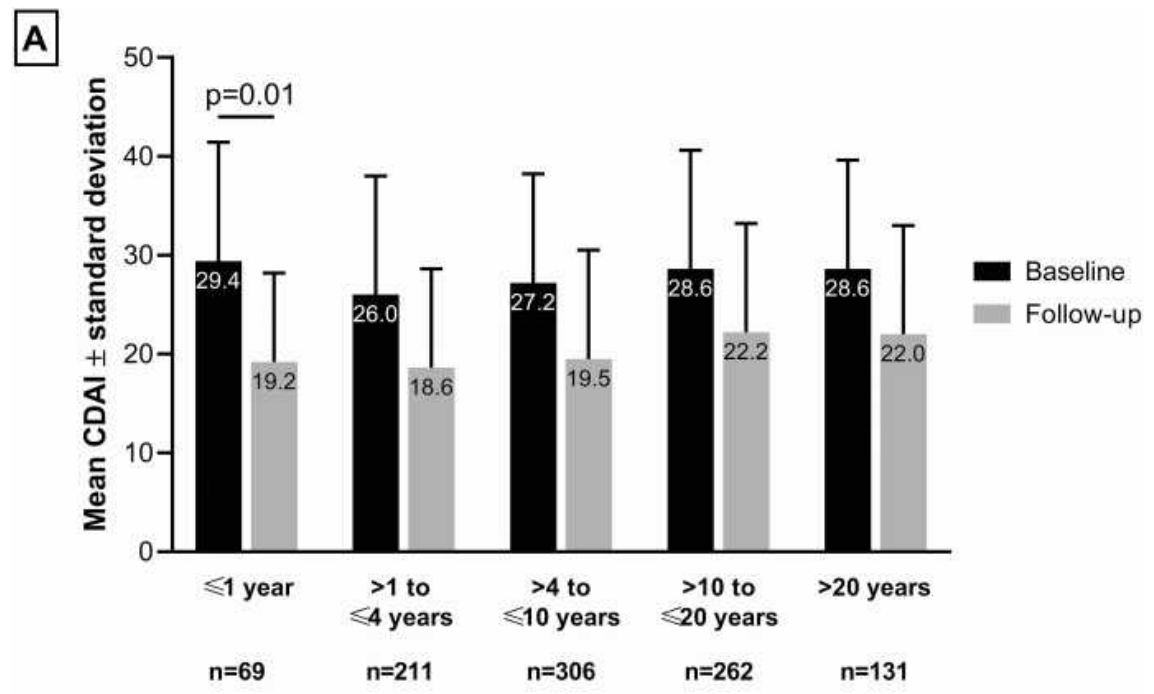

Duration of RA prior to initiation of etanercept treatment

B

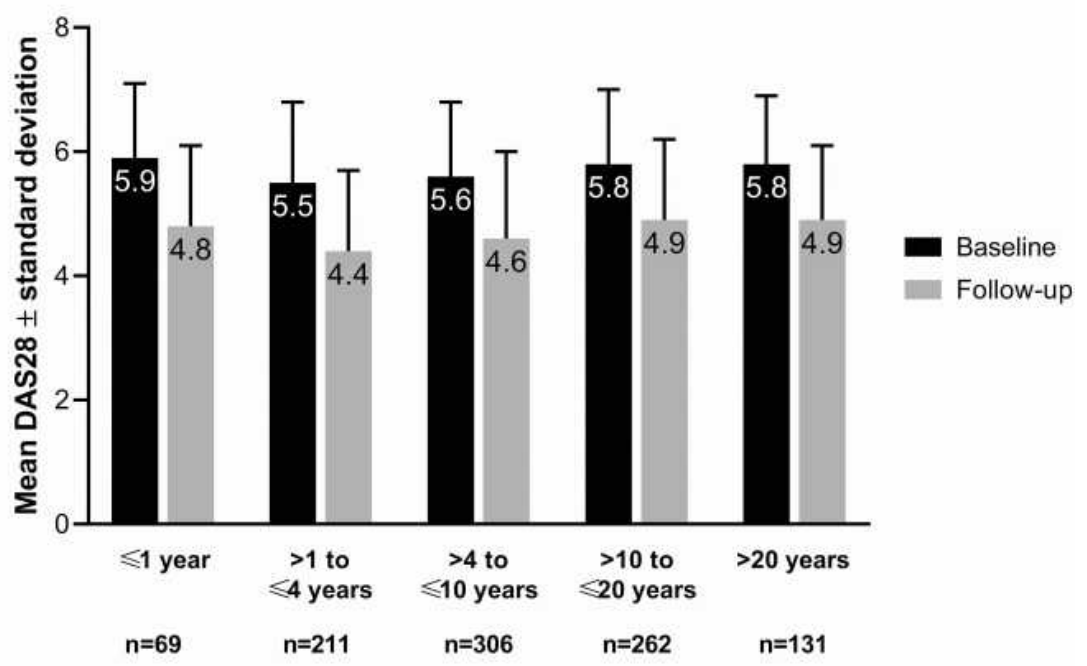

Duration of RA prior to initiation of etanercept treatment

Figure 2 Change from baseline in (A) CDAI and (B) DAS28 in Iraqi patients stratified according to the duration of RA prior to etanercept initiation. Abbreviations: CDAI, Clinical Disease Activity Index; DAS28, 28-joint Disease Activity Score.

DMARD such as etanercept among health care professionals in Iraq should help to improve outcomes for Iraqi patients with this chronic, debilitating disease.

\section{Acknowledgments}

Medical writing support was provided by David Wateridge, $\mathrm{PhD}$, of Engage Scientific Solutions and was funded by Pfizer. The data described in this article were previously reported in the form of an abstract and poster presentation at the ACR Convergence virtual meeting in November, 2020.

\section{Author Contributions}

All authors made substantial contributions to conception and design, acquisition of data, or analysis and interpretation of data; took part in drafting the article or revising it critically for important intellectual content; agreed to submit to the current journal; gave final approval of the version to be published; and agreed to be accountable for all aspects of the work.

\section{Funding}

There is no funding to report. 


\section{Disclosure}

NA-A, FG, DY, FAD, and YAH declare no conflict of interest. NS and AA are employees of Pfizer and hold stock and/or stock options with Pfizer.

\section{References}

1. Finckh A, Liang MH, van Herckenrode CM, de Pablo P. Long-term impact of early treatment on radiographic progression in rheumatoid arthritis: a meta-analysis. Arthritis Rheum. 2006;55(6):864-872. doi:10.1002/art.22353

2. Kyburz D, Gabay C, Michel BA, Finckh A. physicians of SCQM-RA. The long-term impact of early treatment of rheumatoid arthritis on radiographic progression: a population-based cohort study. Rheumatology. 2011;50(6):1106-1110. doi:10.1093/rheumatology/ keq424

3. van Nies JA, Krabben A, Schoones JW, Huizinga TW, Kloppenburg M, van der Helm-van Mil AH. What is the evidence for the presence of a therapeutic window of opportunity in rheumatoid arthritis? A systematic literature review. Ann Rheum Dis. 2014;73 (5):861-870. doi:10.1136/annrheumdis-2012-203130
4. Smolen JS, Landewé RBM, Bijlsma JWJ, et al. EULAR recommendations for the management of rheumatoid arthritis with synthetic and biological disease-modifying antirheumatic drugs: 2019 update. Ann Rheum Dis. 2020;79(6):685-699. doi:10.1136/annrheumdis-2019216655

5. Taylor PC, Feldmann M. Anti-TNF biologic agents: still the therapy of choice for rheumatoid arthritis. Nat Rev Rheumatol. 2009;5 (10):578-582. doi:10.1038/nrrheum.2009.181

6. Almoallim HM, Alharbi LA. Rheumatoid arthritis in Saudi Arabia. Saudi Med J. 2014;35(12):1442-1454.

7. Al Saleh J, Ragab G, Nash P, et al. Rheumatoid arthritis in the Middle East and Africa: are we any closer to optimising its management? Clin Rheumatol. 2015;34(1):1-8. doi:10.1007/s10067-014-2818-5

8. Hussain W, Noorwali A, Janoudi N, et al. From symptoms to diagnosis: an observational study of the journey of rheumatoid arthritis patients in Saudi Arabia. Oman Med J. 2016;31(1):29-34. doi:10.5001/omj.2016.06

9. Hammoudeh M, Al Awadhi A, Hasan EH, Akhlaghi M, Ahmadzadeh A, Sadeghi Abdollahi B. Safety, tolerability, and efficacy of tocilizumab in rheumatoid arthritis: an open-label phase 4 study in patients from the Middle East. Int $J$ Rheumatol. 2015;2015:975028. doi:10.1155/2015/975028
Open Access Rheumatology: Research and Reviews

\section{Publish your work in this journal}

Open Access Rheumatology Research and Reviews is an international, peer-reviewed, open access journal publishing original research, reports, editorials, reviews and commentaries on all aspects of clinical and experimental rheumatology in the clinic and laboratory including the following topics: Pathology, pathophysiology of rheumatological diseases; Investigation, treatment and management

\section{Dovepress}

of rheumatological diseases; Clinical trials and novel pharmacological approaches for the treatment of rheumatological disorders. The manuscript management system is completely online and includes a very quick and fair peer-review system, which is all easy to use. Visit http://www.dovepress.com/testimonials.php to read real quotes from published authors. 